\title{
Study of Lipid Profile in Patients of Polycystic Ovarian Syndrome Before and After Metformin Therapy
}

\author{
Geetika Singh ${ }^{1}$, Nishat Afroz ${ }^{1}$, Noora Saeed ${ }^{1 *}$, Sheelu Shafiq Siddiqi ${ }^{2}$, Aaliya Ehsan ${ }^{3}$ and Mohd Rafey ${ }^{3}$ \\ ${ }^{1}$ Department of Pathology, JNMCH, AMU, Aligarh, U.P, India \\ ${ }^{2}$ Rajiv Gandhi Centre for Diabetes and Endocrinology, JNMCH, AMU, Aligarh, U.P, India \\ ${ }^{3}$ Department of Pathology, JNMCH, AMU, Aligarh, U.P, India
}

\begin{abstract}
Background: Polycystic ovary syndrome (PCOS) is the most common metabolic and endocrine disorders affecting 5-10\% of women in their reproductive age.

Objective:To evaluate the effect of metformin on serum lipid profile in patients of polycystic ovary syndrome.

Methods: An observational prospective study conducted at Rajiv Gandhi Centre for Diabetes and Endocrinology and Department of Pathology,JN Medical College AMU from December 2012 to November 2015. All PCOS women were randomized into two group; one group were received Metformin therapy for 6 months duration and the other group were kept on placebo and followed for the same period. All studied women were subjected to measurement of overnight fasting lipid profile which included total cholesterol,triglycerides, high density lipoprotein cholesterol and low density lipoprotein cholesterol.
\end{abstract}

Results: For women who received Metformin, there was significant improvement in the biochemical profile with a significant $\mathrm{p}$ value of $<0.001$.

Conclusion: Metformin have been shown to improve the lipid profiles leading to increase in the high density lipoprotein cholesterol, the main predictive of cardiovascular disease in the women with polycystic ovary syndrome.

Keywords: Metformin, Polycystic Ovary Syndrome, Lipid Profile.

\section{Introduction}

Polycystic ovarian syndrome (PCOS) is the most common metabolic and endocrine disorders affecting $5-10 \%$ of women in their reproductive age ${ }^{[1]}$ and is a leading cause of infertility. It's main features are lack of regular ovulation and excessive amount or effect of androgenic hormones. ${ }^{[2,3]}$ While the causes of PCOS are still unknown, insulin resistance secondary to obesity is strongly correlated to this syndrome..$^{[4]}$ Obesity increases hyperandrogenism, hirsutism, and infertility and pregnancy complications both independently and by exacerbating PCOS. In general population, obesity and insulin resistance further increase type 2 diabetes (DM2) and cardiovascular disease (CVD). Likewise, in PCOS obesity worsens insulin resistanceand exacerbates reproductive and metabolic features. ${ }^{[5]}$ Furthermore, women with PCOS have increased risk factors for DM2 and CVD,increased impaired glucose tolerance (IGT), DM2 and potentially increased CVD. ${ }^{[6]}$

Metformin, a biguanide antihyperglycemic drug has been shown to have a beneficial effect on circulating lipid level by decreasing the plasma level of triglycerides and total LDL cholesterol and increasing the level of HDL cholesterol independently of the improvement of glycemic control. ${ }^{[7]}$ However there are only a few studies specifically concerning the effect of Metformin therapy on lipid profile in women with PCOS. ${ }^{[8]}$ Moreover Metformin is offered as first line treatment to clomiphene resistant women with PCOS. ${ }^{[9]}$

\section{Material and Methods}

This observational study included 98 patients of Polycystic Ovarian Syndrome (PCOS) in the reproductive age group, attending the outpatient services of the Rajiv Gandhi Centre for Diabetes and Endocrinology and Department of Pathology, JN Medical College AMU. The cases were studied prospectively from December 2012 to November 2015. Informed consent was taken from each patient and the study design was approved by institution review committee. Metformin form one of the treatment approaches for PCOS and previous studies ${ }^{[10]}$ suggest its therapeutic effect in control of the syndrome. Thus this study was performed in order to evaluate the therapeutic effect ofmetformin as treatment for PCOS. 
Inclusion Criteria of the Study and Placebo Groups: Forty nine diagnosed cases of Polycystic Ovarain Syndrome were randomly selected who had already been tested for fasting blood sugar (FBS) serum levels of Luteinizing hormone (LH), Folllicle Stimulating Hormone (FSH), LH/ $\mathrm{FSH}$, testosterone and dehydro-epiandrosterone sulphate (DHEAS) and selected for metformin treatment were included in the study group.

Similarly for placebo group age and weight matched 49 patients of PCOSwere included in the placebo group and were selected for placebo drugs

Exclusion Criteria; Women with prior history of glucose intolerance (including gestational diabetes or type 2 Diabetes mellitus, late onset congenital adrenal hyperplasia cushing's syndrome, hypothyroidism, hyperthyroidism or patients taking medications to alter the hormonal profile were excluded both from study and placebo group.

\section{Material and Method}

After initial clinical assessment, all women who fulfilled the inclusion criteria were explained respective methodology of our study and an informed consent was obtained before the study. Pre-therapy serum lipid profile, thyroid function tests and serum prolactin levels were assessed besides complete history and detailed examination for each patient. The serum of patient was collected after an overnight fast of $10-12 \mathrm{~h}$, separated after centrifugation into two parts. The blood samples were centrifuged at $3000 \mathrm{rpm}$ for 5 minutes, it was essential to ensure that the serum did not hemolyse. The clean serums were transferred to clean plastic tubes by micropipette. The tubeswere topped by plastic stoppers and stored at $-200 \mathrm{C}$ in order to avoid variation, till the time of analysis. In all tests internal quality control tests was performed to detect the accuracy of the results.

Out of the total number of 98 patients of PCOS reported, 49 PCOS patients were administered with Metformin at a dose of 500mg TDS for a period of 6 months while rest 49 PCOS females were prescribed a placebo drug for
6 months. Both the groups were asked to follow same dietary and exercise regime during the study period. After completion of treatment, all the biochemical parameters i.e serum cholesterol, triglyceride, high density lipoproteincholesterol and low density lipoprotein-cholesterol were analyzed and compared with their pre-intervention values in study and placebo group respectively.

\section{Results}

The mean age in the study and placebo group was 27.88 and 28.06 respectively and the age difference between the two groups was found to be insignificant ( $p$-value $=0.87$ ), while the least common age group in both the groups were 36-40 years.(Table 1) (Figure 1)

Regarding the BMI, out of 98 patients a total of $37(37.7 \%)$ patients were overweight with BMI $>25 \mathrm{~kg} / \mathrm{m}^{2}$, while 25 $(25.5 \%)$ patients were obese with BMI $>30 \mathrm{~kg} / \mathrm{m}^{2}$. So a total of 62 cases $(63.3 \%)$ were overweight and obese. (Table 2)

In patients of PCOS the most common complaint was infertility which was seen in $88.7 \%$, followed by obesity which was noted in $63.2 \%$ of the patients. (Table 3 )

Assessment of preintervention lipid profile in 49 PCOS patients of the study group revealed dyslipidemia in form of hypercholesterolemia in $43(87.7 \%)$ cases, high LDL-C in $14(28.5 \%)$ cases and hypertriglyceridemia in $37(75.5 \%)$ patients. Mean serum HDL level was $37.90 \pm 5.452 \mathrm{mg} / \mathrm{dl}$. After 6 months of Metformin treatment the altered lipid profile in study group patients came down to normal level in $31(63.3 \%)$ of patients and the decline in serum levels of total cholesterol, LDL-C and triglyceride was found to be highly significant $(\mathrm{p}<0.001)$, simultaneously at the same time there was also significant rise in serum HDL-C from $38.16 \pm 4.838$ to $55.62 \pm 3.619$, when compared to preintervention levels and to placebo group patients who had either insignificant or minimal alteration in their lipid profile after 6 months of followup (TABLE 4).

Fasting plasma glucose showed improvement after metformin treatment but it was not significant $(p$-value $=0.3$ )

Table 1: Age frequency of the PCOS patients in study and placebo group.

\begin{tabular}{|c|c|c|}
\hline Age in years & STUDY GROUP (\%) & PLACEBO GROUP(\%) \\
\hline $20-25$ & $17(34.7)$ & $16(32.7)$ \\
\hline $26-30$ & $18(36.7)$ & $19(38.7)$ \\
\hline $31-35$ & $09(18.4)$ & $11(22.4)$ \\
\hline $36-40$ & $05(10.2)$ & $03(6.2)$ \\
\hline TOTAL & $\mathbf{4 9 ( 1 0 0 )}$ & $\mathbf{4 9 ( 1 0 0 )}$ \\
\hline
\end{tabular}


Table 2: Body mass index frequency of the patients in study and placebo groups.

\begin{tabular}{|l|c|c|}
\hline BMI $\left(\mathrm{kg} / \mathrm{m}^{2}\right)$ & STUDY GROUP (\%) & PLACEBO GROUP (\%) \\
\hline Underweight $<18.5$ & 00 & $15(30.6)$ \\
\hline Normal weight $(18.5-24.99)$ & $21(42.8 \%)$ & $21(42.8)$ \\
\hline Overweight $(25-29.99)$ & $16(32.6)$ & $13(26.5)$ \\
\hline Obese $>30$ & $12(24.4)$ & 49 \\
\hline Total & 49 & \\
\hline
\end{tabular}

Table 3: The clinical presentations of patients of PCOS.

\begin{tabular}{|l|c|}
\hline Symptoms & No. Of patients (\%) \\
\hline Infertility & $87(88.7)$ \\
\hline Overweight/ obesity & $62(63.2)$ \\
\hline Irregular mensturation & $38(38.7)$ \\
\hline Hirsutism & $21(21.4)$ \\
\hline
\end{tabular}

TABLE 4: Lipid Profile Comparison IN The Study and Placebo Group

\begin{tabular}{|c|c|c|c|c|c|c|}
\hline \multicolumn{4}{|c|}{ Study group } & \multicolumn{3}{|c|}{ Placebo group } \\
\hline Variable (mg/dl) & Pre-t/t & Post-t/t & $p$-value & Pre-t/t & Post-t/t & $p$-value \\
\hline total chol & $323.82 \pm 64.845$ & $170.40 \pm 14.058$ & $<0.001$ & $274.46 \pm 70.225$ & $275.50 \pm 70.243$ & 0.26 \\
\hline LDL-C & $308.98 \pm 64.180$ & $172.96 \pm 14.144$ & $<0.001$ & $249.32 \pm 77.158$ & $249.84 \pm 77.404$ & 0.477 \\
\hline TG & $293.66 \pm 52.757$ & $167.54 \pm 12.216$ & $<0.001$ & $251.22 \pm 66.259$ & $251.46 \pm 66.138$ & 0.855 \\
\hline HDL-C & $38.16 \pm 4.838$ & $55.62 \pm 3.619$ & $<0.001$ & $37.90 \pm 5.452$ & $38.38 \pm 4.085$ & 0.394 \\
\hline
\end{tabular}

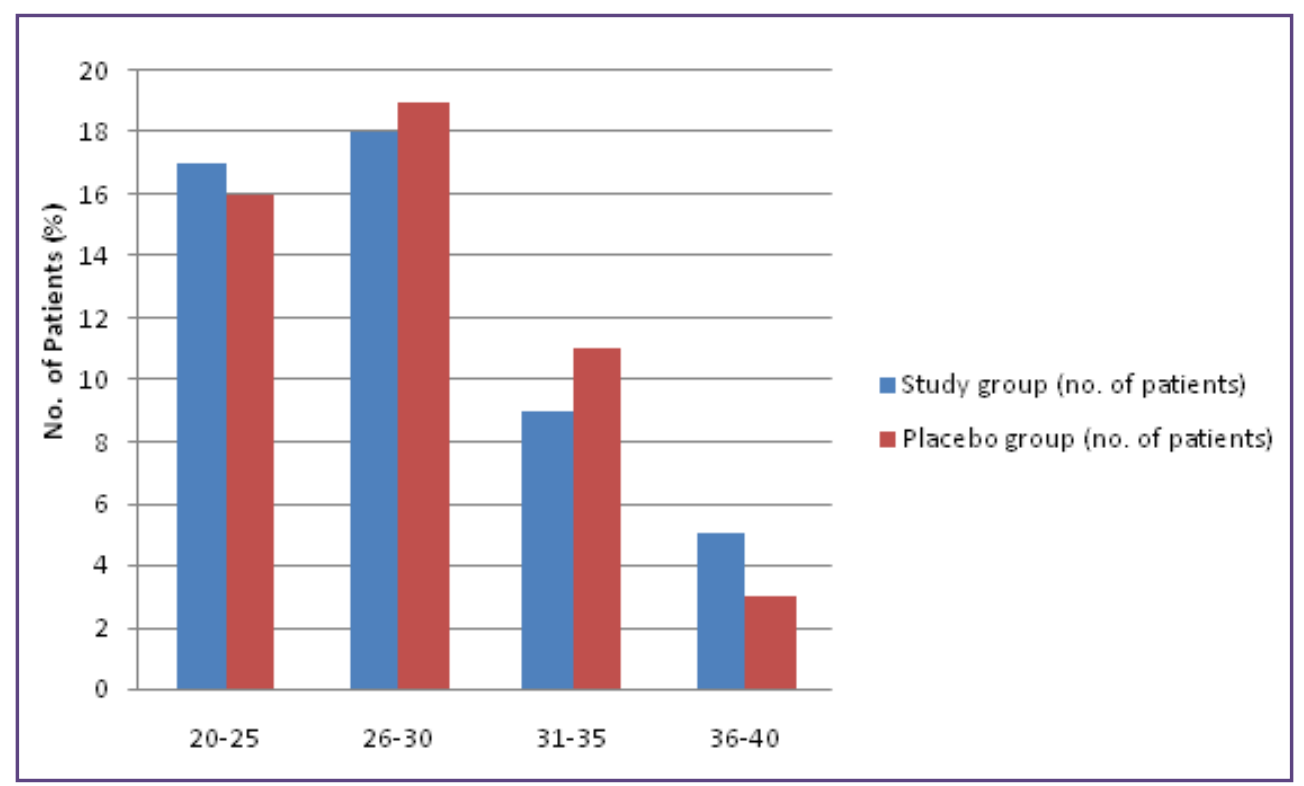

Fig. 1: Agewise distribution of PCOS patients.

\section{Discussion}

PCOS has been a subject of research and debate over past six decades. Insulin resistance accompanied by compensatory hyperinsulinemia is a common feature of PCOS and both obese and non-obese women with the syndrome are more insulin resistant and hyperinsulinemic than age and weight matched normal women. ${ }^{[11]}$ Insulin resistance in muscles and adipose tissues increases plasma free fatty acid (FFA) and insulin concentration, that stimulate synthesis and secretion of VLDL in the liver resulting in hypertriglyceridemia, which in turn enhances post-prandial accumulation of lipoproteins(LDL,VLDL) in plasma with lowering of HDL cholesterol. ${ }^{[12]}$ Hyperinsulinemia plays a pathogenetic role in PCOS cases by increasing ovarian androgen production and decreasing the serum sex hormone binding globulin concentration. ${ }^{[13]}$ 
Almost $70-80 \%$ patients of PCOS are obese. Similarly in our study, almost $64 \%$ cases were overweight and obese with BMI $>25$. Obesity is associated with insulin resistance which is a well known fact by many studies, however patients of PCOS have much more insulin resistance than that of obese women in the general population. ${ }^{[14]}$

The current study shows that a total of 43 out of 49 PCOS patients in study group had high total cholesterol (TC). The fall in TC was significant $(\mathrm{p}<0.001)$ when compared to placebo group after 6 months of Metformin treatment. Similarly LDL-C and triglyceride were also raised in 22 (44.8\%) and 46(93.8\%) of cases respectively.These two parameters also showed a significant fall after Metformin treatment $(\mathrm{p}<0.001)$, when compared to placebo group after Metformin treatment. HDL-C rose significantly from mean level of $38.16 \pm 4.838$ to $55.6 \pm 3.619 \mathrm{mg} / \mathrm{dl}$ after Metformin treatment $(p<0.001)$ when compared to placebo group. The current study results of positive effect of Metformin treatment on lipid profiles in PCOS patients was also corresponding with the results obtained by Karimzadeh et al (2007) and Bratati et al. (2010).Other studies done by Moghetti et al and Velazquez et al showed only a negligible or no effect on serum lipids in women with PCOS.These discrepancies in the result among researches could be explained by difference in the studied population or by shorter duration of treatment. ${ }^{[15]}$ It has been found by some studies done by Morin-papunen et al and Glueck et al that the beneficial effect of Metformin on serum lipid is related to the duration and the dosage of the treatment.

\section{Conclusion}

Metformin- an insulin sensitizer has been shown to improve the serum lipid profiles leading to increase the HDL cholesterol, the main predictive of cardio vascular disease in the women with PCOS.

\section{References}

1. Ehrmann DA. Polycystic ovary syndrome. N Engl J Med 2005; 352: 1223-36.

2. Al-Inany H, Johnson N. Drugs for anovulatory infertility in polycystic ovary syndrome. BMJ 2006; 332:1461-62.

3. Michelmorek, Balen AH, Dunger DB, Vezelymp.Poycystic ovaries and associated clinical and biochemical features in young women.Clinendocrinol(Oxford).1999;51:779-86.
4. Futterweit W, Mechanick JI. Polycystic ovarian disease: etiology diagnosis, and treatment. ComprTher. 1988;14:12-20.

5. Dahlgren E, Janson PO, Johansson S, et al. Polycystic ovary syndrome and risk for myocardial infarction. ActaObstetGynecolScand 1992;71:599-603.

6. Hopkinson Z, Satter N, Fleming R et al. Polycystic ovarian syndrome: the metabolic syndrome comes to gynaecology, BMJ 1998;317:329-32.

7. Kiddy DS, Sharp PS, White DM, et al. Differences in clinical and endocrine features between obese and non-obese subjects with polycystic ovary syndrome: an analysis of 263 consecutive cases.ClinEndocrinol (Oxf) 1990; 32:213-20.

8. Shaw LJ, BaireyMerz CN, Azziz R, et al. Postmenopausal women with a history of irregular menses and elevated androgen measurements at high risk for worsening cardiovascular event-free survival: results from the National Institutes of Health-National Heart, Lung, and Blood Institute sponsored Women's Ischemia Syndrome Evaluation. J ClinEndocrinolMetab 2008, 93:1276-1284.

9. Messaoudi S, Rongen GA, Riksen, "The cardioprotective effects of metformin". Current Opinion in Lipidology 2011;22: 445-53.

10. Dunaif A, Insulin resistance and polycystic ovary syndrome: mechanism and implication for pathogenesis. Endocrine Review 1997; 18: 774-800.

11. Bengtsson C, Bjorkelund C, Lapidusal, Lissnerl. Association of serum lipid concentration and obesity with mortality in women. Twenty years fellow up of participants in prospective population study in Gothenbrg, sweden. Br Med J 1993:1385-88.

12. Zawadski JK, Dunaif A . Diagnostic criteria for polycystic ovary syndrome. Towards a rational approach in: polycystic ovary syndrome. 1992:377-84.

13. Jacobs HS. Polycystic ovaries and polycystic ovary syndrome.GynecolEndocrinol 1987;1:113-31.

14. Fadhil AZ, Al-Dahhan F. The Effect of Metformin on Serum Lipids in Overweight and Obese Patients with Polycystic Ovary Syndrome.The Iraqi Postgraduate Medical Journal. 2014;13:3.

15. Lobo RA, Carmina E. The importance of diagnosing the polycystic ovary syndrome. Ann intern Med 2002;17:2495-99.

*Corresponding author:

Noora Saeed, Senior resident, department of pathology, JNMCH, AMU, ALIGARH, U.P- 202002(India)

Phone: +91 9997240844

Email: dr.noorasaeed@gmail.com

Financial or other Competing Interests: None.

Date of Submission : 26.12.2016

Date of Acceptance : 11.06.2017

Date of Publication : 31.08.2017 